\title{
El estado emocional y el rendimiento académico una aproximación a la realidad actual
}

Emotional state and academic performance. An approach to the current

$$
\text { reality }
$$

1 Emily Estefania Moreano Núñez

(iD) https://orcid.org/0000-0002-9133-3649

Universidad Técnica de Ambato, Facultad de Ciencias de la Educación, Carrera de

Psicopedagogía. Ambato, Ecuador.

emoreano9751@uta.edu.ec

2 Lenin Fabián Saltos Salazar

Universidad Técnica de Ambato, Facultad de Ciencias de la Educación, Carrera de

Psicopedagogía. Ambato, Ecuador.

lf.saltos@uta.edu.ec

3 Wilma Lorena Gavilanes López

Universidad Técnica de Ambato, Facultad de Ciencias de la Educación, Carrera de

Psicopedagogía. Ambato, Ecuador.

wilmalgavilanes1@uta.edu.ec

4 Jorge Rodrigo Andrade Alban

Universidad Técnica de Ambato, Facultad de Ciencias de la Educación, Carrera de

Psicopedagogía. Ambato, Ecuador.

jr.andrade@uta.edu.ec

Artículo de Investigación Científica y Tecnológica

Enviado: 24/12/2021

Revisado: 29/12/2021

Aceptado: $12 / 01 / 2022$

Publicado:08/03/2023

DOI: https://doi.org/10.33262/concienciadigital.v6i1.4.2012

Cítese: Moreano Núñez, E. E., Saltos Salazar, L. F., Gavilanes López, W. L., \& Andrade Alban, J. R. (2023). El estado emocional y el rendimiento académico una aproximación a la realidad actual. $\quad$ ConcienciaDigital, $\quad 6(1.4), \quad 524-550$. https://doi.org/10.33262/concienciadigital.v6i1.4.2012

CONCIENCIA DIGITAL, es una Revista Multidisciplinar, Trimestral, que se publicará en soporte electrónico tiene como misión contribuir a la formación de profesionales competentes con visión humanística y crítica que sean capaces de exponer sus resultados investigativos y científicos en la misma medida que se promueva mediante su intervención cambios positivos en la sociedad. https://concienciadigital.org

La revista es editada por la Editorial Ciencia Digital (Editorial de prestigio registrada en la Cámara Ecuatoriana de Libro con No de Afiliación 663) www.celibro.org.ec 
Palabras claves:

estado emocional, rendimiento académico, emociones, correlacional.
Keywords:

emotional state, academic performance, emotions, correlational
Resumen

Introducción. Los estados emocionales están relacionados fundamentalmente con procesos cognitivos como la atención, la percepción, la memoria y el aprendizaje, la importancia de su inserción en el contexto educativo es esencial, con el fin de proponer a los estudiantes, alternativas y estrategias para que la modulación de las emociones no afecte el rendimiento académico. Objetivo. Esta investigación tiene como objetivo analizar la relación entre el estado emocional y el rendimiento académico y proponer una alternativa para contribuir al desarrollo de los procesos de reconocimiento y regulación de los estados emocionales, reduciendo el fracaso académico y el bajo rendimiento. La investigación se llevó a cabo en el Colegio Belisario Quevedo de Pujilí. Metodología. Se realizó una investigación de campo, descriptiva, experimental y cuantitativa. El universo total es de 278 alumnos, de los cuales mediante la fórmula de muestreo y el muestreo aleatorio simple dieron como resultado una muestra de 161 alumnos que fueron evaluados mediante el Cuestionario del Test de Eysenck y la Escala de Autoconcepto Académico (EAA). Resultados. Los resultados mostraron un nivel significativo de Inestabilidad Emocional y dificultades en Autoestima, Felicidad, Autonomía, tendencias hacia la Depresión, Ansiedad, Obsesividad, Dependencia, Hipocondría y Sentimiento de Culpa; En el caso del Rendimiento Académico: el Rendimiento Académico está presente, así como el Autoconcepto Académico, la Autoeficacia es considerada como baja. Conclusión. Existe una relación directa con tendencia fuerte - moderada entre la autoestima, la ansiedad y el rendimiento académico; la ansiedad y la autoeficacia y la culpa y el autoconcepto académico. Esto demuestra que el estado emocional afecta significativamente al rendimiento académico.

\section{Abstract}

Introduction. Emotional states are fundamentally related to cognitive processes such as attention, perception, memory and learning, these increases the importance of their insertion in educational context, to propose to students, alternatives, and strategies for the modulation of emotions do not affect academic performance. Objective. This research aims to analyze the relationship between emotional state and academic performance 
and purposes an alternative to contribute to the development of processes of recognition and regulation of the emotional states, reducing academic failure and low performance. The research was conducted at the Belisario Quevedo High School, Pujilí. Methodology. A field descriptive, experimental and quantitative research was carried out. The total universe is 278 students of which the sampling formula and simple random sampling resulted in a sample of 161 students who were evaluated using the Eysenck Test Questionnaire and the Academic Self-Concept Scale (EAA). Results. The results showed a significate level of Emotional Instability and difficulties in Self-Esteem, Happiness, Autonomy, tendency to Depression, Anxiety, Obsessiveness, Dependence, Hypochondria and Sense of Guilt; In the case of Academic performance: Academic Performance are present as well as Academic Self-Concept., Self-Efficacy is low and Conclusion. There is a direct relationship with a strong - moderate tendency between self-esteem, anxiety and academic performance, anxiety and self-efficacy and guilt and academic self-concept. This shows that the emotional state significantly affects academic performance.

\section{Introducción}

Las emociones han sido consideradas parte de numerosas y profundas investigaciones. Varios autores las han catalogado como componentes esenciales convirtiéndonos en seres humanos emocionales, capaces de reaccionar ante distintas situaciones. A partir de esto se establece que las acciones del sujeto deben poseer balance entre la emoción y la razón para que de esta manera se produzca un resultado satisfactorio. El estudio de los estados emocionales es sin duda uno de los más complejos debido a su prevalencia de variabilidad en los sujetos y los estudios en curso relacionados con la neurobiología cerebral. El pensar, el sentir y el actuar se centra en cómo el ser humano percibe su ambiente y reacciona activando sus complejos sistemas cerebrales.

Considerando la complejidad del estudio de las emociones, a partir de las investigaciones realizadas en el campo de las neurociencias se han logrado establecer técnicas de neuroimagen funcional como la TEP (Tomografía por emisión de positrones) y RMf (Resonancia Magnética Funcional) mediante estos procesos se ha logrado explorar las distintas áreas cerebrales del ser humano, implicadas en procesos cognitivos. Esto ha permitido establecer un mapeo y tener una idea claramente definida de cómo se producen los procesos mentales y la relación que se establece entre sí (Rovella, 2018). 
Mediante las técnicas mencionadas se lograron definir a las emociones como reacciones psicofisiológicas que se producen dentro de las estructuras del Sistema Límbico y regiones de núcleos corticales y la corteza prefrontal. Particularmente, la amígdala considerada como el centro emocional, que se interrelaciona con procesos cognitivos como el aprendizaje, memoria, atención y percepción (Ripoll, 2014).

Bajo este preámbulo se promulga la importancia de las emociones en todas las esferas del ser humano y sus múltiples contextos tanto familiares como educativos, sociales y personales. Los sujetos por naturaleza mantienen su forma personal de reaccionar ante una determinada situación es así como, de acuerdo con el contexto, a la cultura y la personalidad, se ven reflejados en conductas y comportamientos. Esta premisa es de gran relevancia para entender la principal razón de la importancia del estudio de las emociones en el ámbito educativo, social y personal.

Considerando estos aspectos, se puede declarar que las emociones son el componente esencial que afecta directamente al accionar y a la toma de decisiones, debido a esto, es importante aclarar que, si las emociones son reacciones ante distintas situaciones, la forma en la que se traducen los comportamientos depende de cómo el sujeto reconoce, etiqueta, analiza, regula y expresa sus emociones.

El objetivo principal de entender cómo se configuran los estados emocionales permite establecer procesos por medio de los cuales el individuo reconozca, acepte sus emociones y modifique su comportamiento y pueda alcanzar un óptimo nivel de bienestar en los distintos aspectos de su vida. Partiendo de esta premisa se desarrolló el presente estudio con la visión de guiar y apoyar a los estudiantes en el camino a un óptimo aprendizaje y evitar la prevalencia de dificultades relacionadas con el estado emocional.

\section{Marco Teórico}

Uno de los conceptos que ha tomado énfasis durante los últimos años es la regulación emocional la misma que otorga al individuo la capacidad de modular sus emociones con la finalidad de que estas, al ser negativas, no interfieran con el funcionamiento del individuo en diversos contextos, actividades cotidianas, toma de decisiones, participación y comunicación en sociedad o en entornos como en el hogar, trabajo, escuela o comunidad

\section{Fuente especificada no válida.}

Actualmente, se evidencia un alto índice de problemas de tipo psicosocial y deterioro de la salud mental debido a dificultades relacionadas con el estado emocional. Según datos obtenidos por la UNICEF desde el año 2012 hasta el año en curso, varios programas han sido desarrollados con el fin de fortalecer el desarrollo emocional por medio de la orientación familiar y educativa; sin embargo, debido a la gran influencia de factores 
ambientales, sociales, políticos y económicos, el ser humano se sujeta y adapta a la realidad del desconocimiento y la cultura impuesta por anteriores generaciones.

En el Ecuador, acorde con el último Informe de Salud mental de la población ecuatoriana se determinó que en la actualidad 6 de cada 10 estudiantes experimentaron o presentan un alto riesgo emocional asociado a la pandemia. El 17\% de los estudiantes presentó algún tipo de crisis emocional derivada del confinamiento y prevalece la existencia de un riesgo medio al consumo de alcohol. Por otro lado, se determinó que le principal problema de salud mental fueron las somatizaciones, es decir, las distintas emociones traducidas en comportamientos.

Actualmente, la política educativa se centra en una visión global de la educación, tanto en términos de habilidades cognitivas como emocionales, el desarrollo de las relaciones humanas basadas en valores que ayuden a los estudiantes a identificar, comprender y manejar sus emociones es un objetivo que es considerado prioritario por la educación en la actualidad. Por tanto, la educación es una tarea compartida en la que hay una interacción que se enmarca en el reconocimiento de la dignidad de los demás como seres humanos capaces de cambiar y transformar su entorno para ampliar sus propias oportunidades de vida y la vida de los demás (Orellana, 2021).

Consecuentemente, las instituciones educativas se encuentran frente al reto de contribuir a la construcción del individuo de forma integral. Debido a la emergencia sanitaria COVID- 19 y el confinamiento ha existido un deterioro la capacidad de las personas para regular su estado emocional y por ende esto los limita a alcanzar un nivel de rendimiento académico adecuado a los estándares educativos. Es por esto por lo que mediante esta investigación se da a conocer información sumamente relevante para entender cómo se presentan estas dificultades en el estado emocional, cómo modular las emociones y cómo enriquecer el proceso educativo mediante la incorporación de hábitos y técnicas de regulación del estado emocional.

Por otra parte, en octubre de 2020 la UNICEF logró obtener datos esenciales para determinar el estado emocional de niñas, niños y adolescentes en Ecuador. De los encuestados 4 de cada 10 manifestaron sentirse tensos o bajo tensión. Los adolescentes fueron los que demostraron mayores efectos adversos derivados de la emergencia sanitaria. Como afirma González (2020), la salud mental está directamente relacionada con el bienestar emocional, psicológico y social, afecta la forma en la que pensamos, sentimos y actuamos, determina la capacidad en la que podemos manejar el estrés y cómo relacionarnos con las demás personas, siendo la clave para que todos puedan ser funcionales en la sociedad.

Uno de los grupos que requieren mayor atención considerando el estado emocional son los adolescentes, esto debido a que son más vulnerables a padecer dificultades o patrones 
de conducta inestables. La adolescencia presupone un periodo transitorio de gran impacto en la vida de las personas, por consiguiente, en el aspecto emocional existen tendencia hacia la apatía, los estados de ánimo negativos e inestabilidad emocional. Bajo esta premisa es pertinente que sea considerada la implementación de mecanismos de regulación del estado emocional dentro del contexto educativo y familiar ya que fomentan la educación de las emociones (Mónaco, 2017).

La importancia de considerar a la adolescencia como parte de un grupo prioritario en temas de salud mental radica en que esta etapa es un punto de inflexión en el desarrollo de los seres humanos debido a los cambios que se presentan tanto a nivel físico como mental, emocional y social; es fundamental conocer que en esta etapa existe una reorganización afectiva y existencial en la que el sujeto requiere orientación y apoyo oportunos con el fin de prevenir posibles comportamientos disruptivos o incluso trastornos de la personalidad (Güemes-Hidalgo, 2017).

Los estados emocionales están relacionados fundamentalmente con los procesos cognitivos tales como la atención, la percepción, memoria y aprendizaje lo que reivindica la importancia de su inserción dentro del contexto educativo, con el fin de proponer a las estudiantes alternativas, estrategias y medios para la modulación de las emociones y que estas al ser negativas, no afecten al desempeño académico y la adquisición de conocimientos. Por otro lado, dentro del contexto educativo, las emociones conducen el aprendizaje, son determinantes en la toma de decisiones, influyen en la creatividad, en las relaciones sociales y en la salud.

Para determinar cómo se procesan y se producen los estados emocionales, se proponen los enfoques principales: Enfoque Procesual Sistémico y el Enfoque Perceptual. Desde el enfoque Procesual la personalidad se construye mediante un proceso dinámico, en el que intervienen varios factores y está en evolución permanente, este a su vez, empieza a consolidarse desde las estructuras más simples hasta que por medio de la experiencia se transforman en estructuras complejas. De esta forma se determina que la personalidad es cambiante y contempla varios procesos y elementos tales como la identidad, la actitud hacía él mismo, la conducta inter e intrapersonal, la forma de ver y apreciar el mundo. Todo esto depende de situaciones externas o internas de la persona para que se produzcan los estados emocionales (Guajardo, 2015).

Por otra parte, el enfoque perceptual propuesto por William James y Carl G. Lange hace énfasis en el objeto y es conocida como la Teoría de la Sensación. Se determina entonces que la percepción de un objeto, hecho, situación o evento determinado tiende a despertar un estado emocional y este a su vez, produce una acción o manifestación mediante cambios corporales. Bajo este criterio, se plantea la estructura en la que el objeto emotivamente significativo produce distintas percepciones de este, posteriormente la 
emoción es producida y esta se expresa por medio de cambios corporales en lo que se demuestra la conducta abierta y observable (Melamed, 2016).

Acorde con García-Cerda (2016), el estado emocional puede ser considerado de acuerdo con dos tipos: Estabilidad e Inestabilidad emocional. En los estudios realizados por el anteriormente citado autor, se logró deducir que la estabilidad emocional describe una disposición estable en forma relativa y guarda relación con la capacidad de mantener el equilibro de forma que este sea automático y recurrente sin influencia externa, aunque se enfrente a una situación desfavorable (Gracia, 2016). Estos hallazgos permitieron determinar que la estabilidad emocional es un rasgo de la personalidad y que tiene dos dimensiones: el umbral de alteración emocional (capacidad de experimentar estados emocionales alterados y la resiliencia emocional (capacidad para recuperarse cuando la persona experimenta situaciones de tipo negativo y logra volver a la estabilidad); también se determinó que existe variabilidad de acuerdo al género, es así que se determinó que las mujeres poseen un umbral de alteración emocional más bajo y menor resiliencia emocional que los hombres.

Por otro lado, se considera que el segundo tipo: Inestabilidad emocional es considerada como un rasgo de personalidad cuya característica principal es que la persona presenta una variación de sentimientos y estados emocionales, así como altibajos de ánimo sin que existan motivos o causas identificables (Berlanga, 2008).

A partir de este análisis es indispensable reconocer que el estado emocional debe ser considerado para que el proceso de aprendizaje sea efectivo y el rendimiento académico sea adecuado. Partiendo de este aporte, se estipula que el rendimiento no es un componente que debe estudiarse de forma independiente. Según Benítez (citado en Navarro, 2003), el rendimiento no tiene que ver solo con la inteligencia y las habilidades, sino que requiere que se tomen en cuenta factores como el nivel socioeconómico, el alcance de los programas de estudio, la metodología empleada, los conocimientos previos de los estudiantes, el nivel de pensamiento formal, habilidades sociales y cognitivas.

Es de igual importancia considerar que existen condiciones fundamentales para que exista un rendimiento académico satisfactorio, debe ser percibido a partir de su naturaleza multifactorial; considerar que el sujeto posee características propias y debe ser vinculado con otros factores como la motivación, e autocontrol, las destrezas y habilidades sociales y la personalidad de cuyo innatismo se producen los estados emocionales (Obando, 2017).

Para el estudio de las implicaciones de las emociones en el rendimiento académico se destaca el aporte psicopedagógico en el que se determina que el campo de estudio de las emociones ha comenzado a fortalecerse en las últimas décadas. Según los estudios realizados por Gallardo (2016), en la educación se garantiza el desarrollo integral del estudiante desde las distintas esferas del ser humano, con respecto al componente 
emocional establece que debe ser incorporado paulatinamente y debe prever que el sujeto desarrolle aspectos relacionados con el alto y positivo sentimiento sobre sí mismo, desde su propia percepción física, psíquica social y personal, la confianza en sí mismo, actitud de tipo optimista o desenfadada, adecuado control de las emociones de tipo negativo, la serenidad global en cuestiones emocionales, control de la propia voluntad y que todas estas destrezas se produzcan permitiendo el desarrollo intelectual (Gallardo Vázquez, 2016).

El alcance global que ha tenido el estudio de las emociones en los últimos años ha permitido la ejecución de diversas investigaciones. Es así como Pekrund (2017), proponen el análisis de los estados emocionales y el logro académico en su investigación "Achievement emotions and Academic Performance: Longitudinal Models of Reciprocal Effects"; se estableció que existe relación entre emociones y el logro académico. Es así como las emociones de tipo positivo tales como la alegría, orgullo, disfrute son aquellas que de alguna manera predicen el rendimiento académico de forma positiva, es decir que los estudiantes que presentan mayor presencia de emociones positivas son aquellos que obtienen mejores resultados al final del ciclo escolar y puntaje en las evaluaciones.

A su vez, las emociones de tipo negativo como la ira, ansiedad, vergüenza, aburrimiento, desesperanza predicen un bajo rendimiento académico y los estudiantes presentaron bajos puntajes en evaluaciones y calificaciones (Pekrun, 2017).

Los estudios realizados en España fueron relevantes para comprender la Influencia de las emociones sobre el Rendimiento Académico. Es así como los estados emocionales se pueden considerar desde la perspectiva personal de la IE (Inteligencia emocional) y por consiguiente un nivel medio y alto de IE conlleva a un mejor rendimiento académico. Por otro lado, la variable principal que produce bajo rendimiento académico es el miedo. Cabe recalcar que la una de las alternativas de solución considerada es la potencialización de las competencias emocionales con el fin de mejorar los resultados académicos y el proceso de aprendizaje (Pulido \& Herrera, 2017).

El alcance de estas investigaciones se fundamenta en otros estudios realizados por Valiente (2012), y otros destacan en su artículo "Linking Students Emotions and Academic Achievement: When and why emotions matter" destacan hallazgos sobre el rol fundamental de las emociones positivas o negativas para el rendimiento académico. Se determina entonces que las emociones son mediadores de los procesos cognitivos y de la motivación, el potencial de control y las relaciones en el aula.

En los últimos años se han realizado distintos programas y proyectos enfocados en el mejoramiento de la regulación emocional. Es así como a nivel internacional la Universidad de Yale crea el Centro para la Inteligencia Emocional, la misma que es una unidad creada para la investigación y capacitación continuas en lo que respecta a 
inteligencia emocional. El propósito fundamental de este centro en brindar apoyo a las instituciones de educación a entender el valor de las emociones y reeducar en materia de las habilidades para la inteligencia emocional y así poder construir climas sustentables de emociones positivas que contribuyan al aprendizaje.

Mediante un modelo denominado SEL (Social and Emotional Learning) desarrollado por la Universidad de Yale, propone a las instituciones, hogares y ambientes laborales una iniciativa en la que participen tanto directivos como educadores y personal para que les permita desarrollar habilidades para el aprendizaje emocional. Por medio de la teoría y la práctica se realizaron distintos estudios que determinaron el impacto dela inteligencia emocional en la vida de todas las personas y los beneficios que aporta a la autoconciencia, autocontrol y autorregulación emocional para la toma de decisiones, la creatividad y el afrontamiento de situaciones desfavorables para los adolescentes propiciando la existencia de un clima escolar adecuado y mejoras en el rendimiento académico de los estudiantes (Brackett, 2020).

Por medio del sustrato investigativo de Yale Center for Emotional Intelligence se probó la pertinencia e impacto de RULER, un acrónico de habilidades para la inteligencia emocional tales como: reconocer, comprender, etiquetar, expresar y regular. Bajo este enfoque se fundamente la Inteligencia emocional. Mediante el modelo planteado se logró capacitar e implementar programas para el desarrollo de habilidades relacionadas con el autocontrol, regulación y aprendizaje emocionales para introducir climas escolares positivos (Hoffmann, 2020).

A nivel nacional en el 2020 se publicó el manual Contención emocional a las familias en situaciones de crisis, el mismo que fue implementado como parte del programa Educando en Familia del Ministerio de Educación. Este manual fue socializado por los DECE (Departamentos de Consejería Estudiantil), como parte de este proceso se proponen talleres con el fin de mejorar el manejo emocional en situaciones de crisis. Este material creado a partir del impacto de la emergencia sanitaria en la salud mental de los niños, niñas, adolescentes y sus familias permitió que se establezcan mecanismos para controlar crisis emocionales dentro del núcleo familiar.

Por último, el estado emocional representa un factor relevante y significativo en el campo de la educación. A partir del reconocimiento y manejo de los estados emocionales se pueden mejorar y transformar significativamente las prácticas educativas de enseñanza y aprendizaje. Si se trabaja a partir de las emociones de forma adecuada, se motivará a los estudiantes al alcance del logro de los aprendizajes.

Es así como es de gran importancia para las personas que forman parte del contexto educativo que reconozcan que los estados emocionales deben ser considerados como fenómenos sociales que son parte de la vida de todos los seres humanos y que por ende 
están presentes en el proceso de enseñanza - aprendizaje. Es por esto que los estados emocionales siempre van a influir significativamente en el logro de los objetivos educativos, en la conducta del estudiante, el dominio de los conocimientos en la disciplina y en lo personal.

Mediante investigaciones recientes, el gran impacto que tiene la dimensión social y emocional en el rendimiento académico es evidente, incluso en la salud, bienestar mental y en el desarrollo de competencias importantes para el aprendizaje, adquisición y consolidación de conocimientos. Por otra parte, es indispensable para el desarrollo de habilidades y destrezas cognitivas tales como la planificación, atención, memoria y metacognición (Silva, 2014).

A partir de esto según la teoría de Schachter \& Singer del año 1962 las emociones son consideradas como elementos importantes para los procesos cognitivos, la cognición como tal y los sentimientos conscientes son considerados como una tendencia a dirigirse hacia situaciones u objetos deseables, esto a su vez, conduce a la acción o aplicación de estrategias mediadas por el componente emocional (Meléndez, 2016).

\section{Metodología}

La presente investigación es de corte mixto cualitativo y cuantitativo. Es decir, posee el sustrato teórico a partir del análisis de distintos conceptos y características previamente descritas y la utilización de estadísticos e instrumentos estandarizados para la recolección de datos objetivos y cuantificables.

Por otra parte, considera la modalidad bibliográfica documental basada en distintas fuentes primarias y secundarias. Al ser realizada bajo los criterios actuales se considera la modalidad de campo de tipo virtual puesto que la información recabada se realizó mediante las distintas plataformas virtuales como Zoom y Google Forms. A su vez presenta niveles a partir del descriptivo puesto que se detalla de manera concisa al fenómeno de estudio estableciendo un marco conceptual. Por otro lado, se considera el nivel correlacional debido a que exístela aplicación de estadígrafos especializados (Pruebas de normalidad, Coeficiente de Correlación de Pearson y Coeficiente de Correlación de Spearman) para determinar y analizar si existe relación entre las variables sujetas el estudio.

La población de estudio fue elegida bajo el criterio de muestreo aleatorio simple, el que determina que todos los participantes tienen la misma oportunidad de ser parte de la investigación. La población total fue de 278 estudiantes de Segundo de Bachillerato de la Unidad Educativa Belisario Quevedo del cantón Pujilí, comprendidos en edades entre 15 a 17 años, el género predominante es femenino. Mediante la fórmula de muestreo se obtuvo finalmente que la muestra es de 161 estudiantes. 
Los datos obtenidos acerca del estado emocional fueron obtenidos a partir de la aplicación, calificación y análisis del Test de Eysenck Cuestionario para medir el nivel inestabilidad - estabilidad emocional (Boyle, 2016), estructurado a partir de 220 preguntas con tres opciones de respuesta Sí, no, ¿indeciso? Este instrumento direccionado a conocer las dificultades del estado emocional explorando dimensiones como: autoestima, depresión, ansiedad, obsesividad, felicidad, hipocondría y sentido de culpa. A partir de la calificación se logró obtener el perfil general de los estudiantes en el que se logra demostrar la tendencia.

Para la evaluación de la segunda variable: rendimiento académico se procedió a la aplicación, calificación e interpretación mediante la escala de autoconcepto académico (EAA) creada por Schmidt (2008), la misma que permite evaluar de forma completa el rendimiento académico y factores como el autoconcepto académico y la autoeficacia; consta de 12 preguntas las mismas que se dividen en factores, a partir de selección múltiple ente Totalmente de acuerdo, De acuerdo, Ni de acuerdo ni en desacuerdo y totalmente en desacuerdo.

Los instrumentos aplicados para el estudio fundamentan su validez y pertinencia en el marco conceptual. Por otro lado, el objetivo primordial de los instrumentos es conocer el impacto del estado emocional en el rendimiento académico mediante la evaluación multifactorial.

Para sistematizar la información se procedió a dividirla en etapas:

$\checkmark$ Primera etapa (Determinación del tema, selección de la población)

$\checkmark$ Segunda etapa: Identificación de los objetivos e hipótesis del estudio.

$\checkmark$ Tercera etapa: Definición de los instrumentos para la recolección de datos.

$\checkmark$ Cuarta etapa: Recolección de datos, aplicación de instrumentos, interpretación y análisis.

$\checkmark$ Quinta etapa: Resultados obtenidos, análisis y discusión.

$\checkmark$ Sexta etapa: Presentación de resultados.

\section{Resultados}

La aplicación de ambos instrumentos permitió obtener los datos concernientes a las dos variables tanto del estado emocional como del rendimiento académico. Por consiguiente, se establecen los siguientes informes de resultados.

Test de Eysenck cuestionario para medir el nivel de inestabilidad-estabilidad emocional

En la tabla 1 se observa que existen siete dimensiones o factores que se evalúan en el test de Eysenck cada uno de los cuales es evaluado de acuerdo con distintas preguntas. A partir de esto tenemos que los puntajes de las dimensiones autoestima, felicidad, ansiedad, 
obsesividad, autonomía, hipocondría y culpa son obtenidas mediante la sumatoria de las preguntas correspondientes y para establecer la calificación general se realiza la fórmula de promedio por cada dimensión.

Tabla 1

Dimensión Autoestima

\begin{tabular}{lll}
\hline \multicolumn{3}{l}{ AUTOESTIMA (Self -esteem) } \\
\hline $1+$ & $71+$ & $141-$ \\
\hline $8-$ & $78-$ & $148+$ \\
\hline $15-$ & $85-$ & $155+$ \\
\hline $22-$ & $92-$ & $162-$ \\
\hline $29+$ & $99-$ & $169-$ \\
\hline $36-$ & $106+$ & $176-$ \\
\hline $43-$ & $113-$ & $183-$ \\
\hline $30-$ & $120-$ & $190-$ \\
\hline $37+$ & $127-$ & $197+$ \\
\hline $64+$ & $134-$ & $204+$
\end{tabular}

Fuente. Datos obtenidos de la aplicación de test en Google Forms

Tabla 2

Dimensión: Felicidad

\begin{tabular}{rrr}
\hline \multicolumn{3}{c}{ FELICIDAD (Happiness) } \\
\hline-2 & -72 & 142 \\
\hline-9 & 79 & -149 \\
\hline 16 & 86 & -156 \\
\hline 23 & -93 & -163 \\
\hline-30 & -100 & -170 \\
\hline 37 & -107 & 177 \\
\hline-44 & -114 & -184 \\
\hline 51 & 121 & -191 \\
\hline-58 & 128 & -198 \\
\hline-65 & -135 & 205 \\
\hline TOTAL & & NN
\end{tabular}

Fuente: Datos obtenidos de la aplicación de test en Google Forms 


\section{DDigital}

\section{Tabla 3}

Dimensión: Ansiedad

\begin{tabular}{rrr}
\hline \multicolumn{3}{c}{ ANSIEDAD (Anxiety) } \\
\hline 3 & 73 & 143 \\
\hline-10 & 80 & 150 \\
\hline-17 & 87 & -157 \\
\hline 24 & -94 & 164 \\
\hline 31 & 101 & 171 \\
\hline 38 & 108 & 178 \\
\hline 45 & 115 & 185 \\
\hline 52 & -122 & 192 \\
\hline 59 & 129 & 199 \\
\hline-66 & 136 & 206 \\
\hline TOTAL & & 0
\end{tabular}

Fuente: Datos obtenidos de la aplicación de test en Google Forms

\section{Tabla 4}

Dimensión: Obsesividad

\begin{tabular}{rrr}
\multicolumn{3}{r}{ OBSESIVIDAD (Obsessive) } \\
\hline 4 & 74 & 144 \\
\hline 11 & 81 & 151 \\
\hline 18 & 88 & 158 \\
\hline 25 & 95 & 165 \\
\hline 32 & 102 & 172 \\
\hline 39 & 109 & 179 \\
\hline 46 & -116 & 186 \\
\hline 53 & -123 & 193 \\
\hline 60 & 130 & 200 \\
\hline 67 & 137 & 207 \\
\hline TOTAL & & $\mathrm{NN}$
\end{tabular}

Fuente: Datos obtenidos de la aplicación de test en Google Forms 


\section{Tabla 5}

Dimensión: Autonomía AUTONOMIA (Autonomy)

\begin{tabular}{rrr}
\hline-5 & 75 & -145 \\
\hline 12 & -82 & -152 \\
\hline 19 & 89 & -159 \\
\hline-26 & 96 & -166 \\
\hline 33 & -103 & -173 \\
\hline-40 & -110 & 180 \\
\hline-47 & -117 & -187 \\
\hline-54 & -124 & -194 \\
\hline 61 & 131 & -201 \\
\hline-68 & 138 & -208 \\
\hline TOTAL & & $\mathrm{NN}$
\end{tabular}

Fuente: Datos obtenidos de la aplicación de test en Google Forms

\section{Tabla 6}

Dimensión: Hipocondría

\begin{tabular}{rrr}
\hline \multicolumn{3}{r}{ HIPOCONDRÍA (Hypochondria) } \\
\hline-6 & 76 & 146 \\
\hline 13 & 83 & 153 \\
\hline 20 & 90 & 160 \\
\hline 27 & 97 & -167 \\
\hline 34 & -104 & 174 \\
\hline 41 & 111 & 181 \\
\hline 48 & 118 & 188 \\
\hline 55 & 125 & 195 \\
\hline 62 & 132 & 202 \\
\hline 69 & 139 & 209 \\
\hline TOTAL & & 0
\end{tabular}

Fuente: Datos obtenidos de la aplicación de test en Google Forms 


\section{Tabla 7}

Análisis global de resultados

\begin{tabular}{ll}
\hline \multicolumn{2}{c}{ Muestra: 161 estudiantes } \\
\hline \multicolumn{2}{c}{ Puntuaciones Generales Obtenidas } \\
\hline Test de Eysenck Cuestionario para medir el nivel de Estabilidad- Inestabilidad emocional \\
\hline DIMESIONES & PUNTUACIÓN \\
\hline AUTOESTIMA & 16 \\
\hline FELICIDAD & 16 \\
\hline ANSIEDAD & 16 \\
\hline OBSESIVIDAD & 15 \\
\hline AUTONOMÍA & 16 \\
\hline HIPOCONDRÍA & 12 \\
\hline SENTIDO DE CULPA & 15
\end{tabular}

Fuente: Datos obtenidos de la aplicación de test en Google Forms

En la tabla 7 se puede observar los puntajes obtenidos por los estudiantes de segundo de bachillerato en cada una de las dimensiones del test. Cada uno de los apartados se califica sobre 30 puntos, el máximo valor a obtenerse. Para analizar los resultados globales es importante entender que cada apartado del instrumento se calificado de acuerdo con los parámetros establecidos en las tablas $1-7$. Mediante la calificación del instrumento, se logra obtener la figura 1, el mismo que proporciona la tendencia global hacia la estabilidad o inestabilidad emocional para su posterior interpretación.

\section{Figura 1}

Perfil Gráfico Test de Eysenck

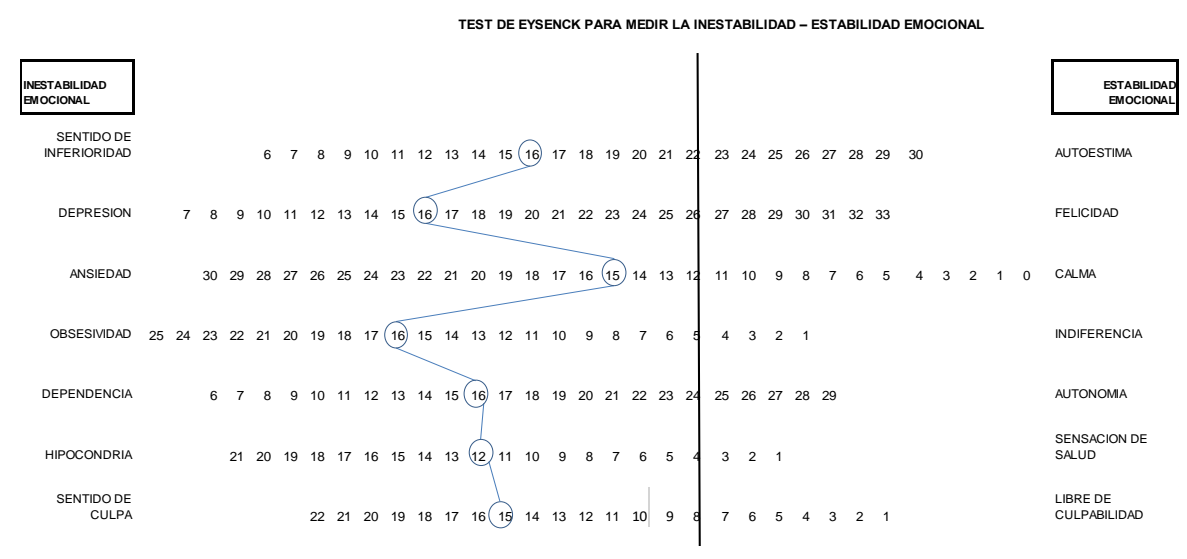

Fuente: Datos obtenidos de la aplicación de test en Google Forms 
Tal como se observa en la figura 1, el perfil permite observar la tendencia hacia la inestabilidad o estabilidad emocional. Los resultados obtenidos determinan que a nivel general los estudiantes presentan el estado emocional de tipo inestable, establecido en una escala menor que 30 en cada dimensión. Evidencia de que existe inestabilidad emocional y tendencia hacia: sentido de inferioridad, depresión. ansiedad, obsesividad, dependencia, hipocondría y sentimientos de culpa. Para detallar adecuadamente las dificultades del estado emocional encontradas se lograron determinar que existen sentimientos de inferioridad, es decir, presencia de patrones de comportamiento que conducen a la persona a poseer baja autoestima e inseguridad. A su vez, la tendencia muestra rasgos de depresión, ansiedad y dependencia, incapacidad de tomar decisiones por cuenta propia, anteponer los deseos de los demás antes que los propios. Por otro lado, existe tendencia a la hipocondría y sentido de culpa. Es importante establecer que los sujetos presentan inestabilidad emocional en un rango moderado.

Informe de resultados Escala de Autoconcepto Académico (EAA)

Tabla 8

Resultados generales escala de autoconcepto académico (EAA) segundo de bachillerato

Curso: Segundo de Bachillerato

Escala de Autoconcepto Académico

Muestra total $=161$ estudiantes

\begin{tabular}{lccc}
\hline & Rendimiento & Autoeficacia & Autoconcepto académico \\
\hline Puntaje bruto & 27 & 24 & 51 \\
\hline Puntaje percentil & 55 & 10 & 65 \\
\hline
\end{tabular}

Fuente: Datos obtenidos de la aplicación de test en Google Forms

Una vez obtenidos los resultados de la aplicación de la Escala de Autoconcepto Académico (EAA), se presenta la tabla 8. Se observan las tres dimensiones del instrumento: Rendimiento, Autoeficacia y Autoconcepto Académico. Cada una de las dimensiones cuentan con un puntaje bruto y un puntaje percentil, los mismos que de acuerdo con la escala de evaluación establece que el puntaje general es de 51 y de 65 respectivamente.

Para establecer un análisis más profundo del instrumento se procede a dividirlo sus respectivas dimensiones: 
Tabla 9

Dimensión: Autoeficacia

\begin{tabular}{lr}
\hline \multicolumn{2}{c}{ Autoeficacia } \\
\hline Muestra & 161 \\
\hline Excelente & $0 \%$ \\
\hline Bueno - Regular & $70,58 \%$ \\
\hline Malo & $29,42 \%$
\end{tabular}

Nota: Datos obtenidos de la aplicación de test en Google Forms

\section{Figura 2}

Dimensión: Autoeficacia

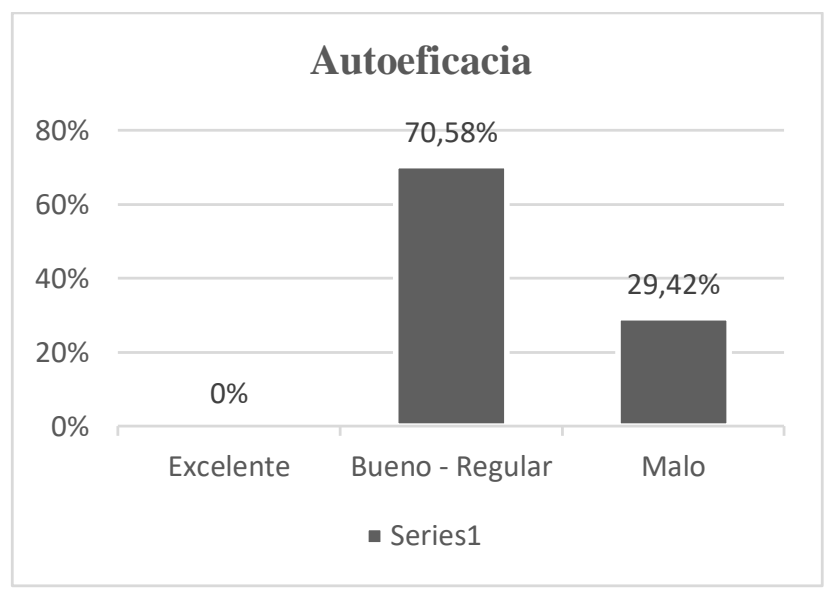

Fuente: Datos obtenidos de la aplicación de test en Google Forms

En la tabla 9 y la figura 2 se puede observar la dimensión de Autoeficacia, los estudiantes obtuvieron una puntuación general de 24 percentil 10, corresponde a un rango medio bajo. Esto demuestra que los estudiantes presentan dificultades en esta dimensión, así como deficiente confianza en las capacidades. Además, el grupo no siempre percibe su capacidad para mantener un rendimiento académico adecuado o en las calificaciones. Cabe recalcar que esta dimensión al ser relacionada con el autoconcepto académico delimita que las dificultades para la autoeficacia pueden ser consecuencia de factores ambientales, familiares o personales. 
Tabla 10

Dimensión: Rendimiento Académico

\begin{tabular}{lr}
\hline \multicolumn{2}{c}{ Rendimiento Académico } \\
\hline Muestra & 161 \\
\hline Excelente & $0 \%$ \\
\hline Bueno -Regular & $79,40 \%$ \\
\hline Malo & $20,60 \%$
\end{tabular}

Nota: Datos obtenidos de la aplicación de test en Google Forms

\section{Figura 3}

Dimensión: Rendimiento Académico

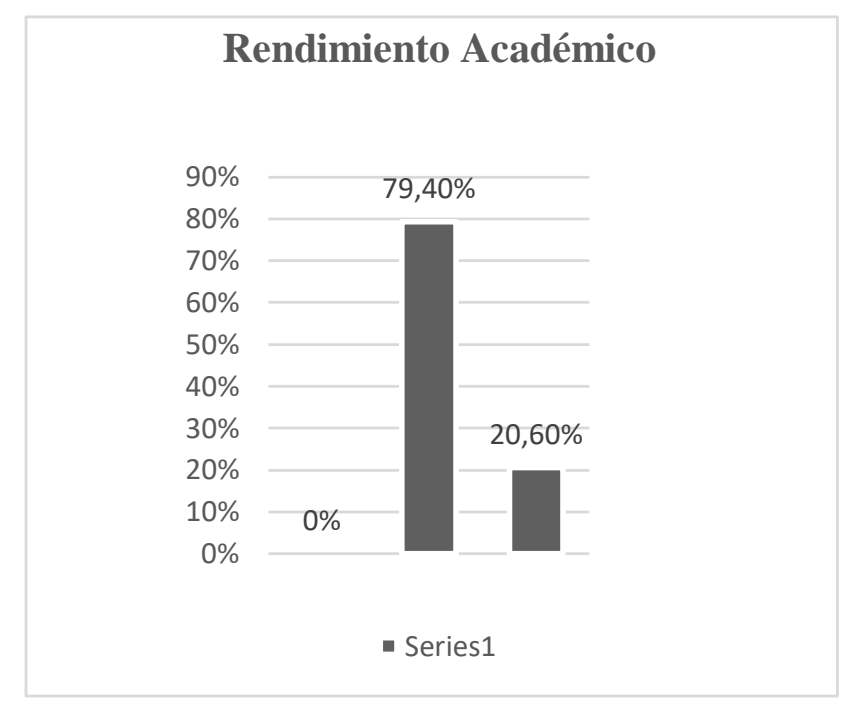

Fuente: Datos obtenidos de la aplicación de test en Google Forms

En la dimensión de Rendimiento Académico, tal como se observa en la tabla 10 y en la figura 3, la puntuación general obtenida es de 27 y percentil 55, correspondiente al rango medio. Los estudiantes no presentan dificultades graves para el aprendizaje, las limitaciones son leves y los estudiantes consideran que no comenten errores significativos o que les cueste estudiar y entender. 
Tabla 11

Dimensión: Autoconcepto Académico

\begin{tabular}{lr}
\hline \multicolumn{2}{c}{ Autoconcepto } \\
\hline Muestra & 161 \\
\hline Excelente & $0 \%$ \\
\hline Bueno - Regular & $77,27 \%$ \\
\hline Malo & $22,73 \%$
\end{tabular}

Fuente: Datos obtenidos de la aplicación de test en Google Forms

\section{Figura 4}

Dimensión: Autoconcepto Académico

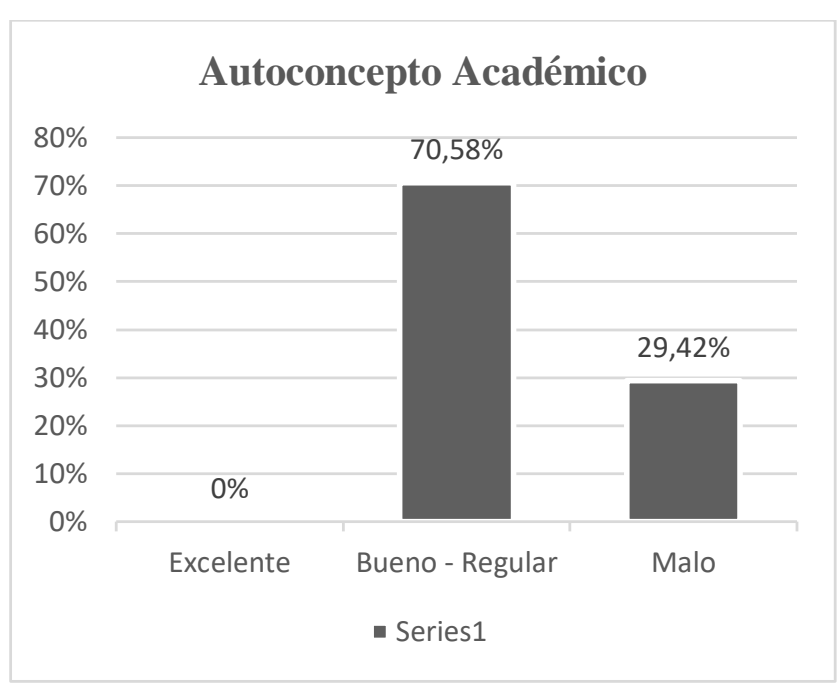

Fuente: Datos obtenidos de la aplicación de test en Google Forms

Tal como se observa en la tabla 11 y en la figura 4, en la dimensión de autoconcepto académico los estudiantes obtuvieron una puntuación bruta de 51 y percentil 61. A partir de la interpretación se estable que los alumnos sí poseen autoconcepto, es decir, lo que piensan de sí mismos, esto prevalece en proporción media y se auto perciben como estudiantes buenos y regulares.

\section{Pruebas de Hipótesis}

Mediante el análisis realizado a las variables de estudio se pretende demostrar si existe relación entre ellas. De esta forma mediante el planteamiento de la hipótesis se obtiene que Ho: El estado emocional no incide en el rendimiento académico de los estudiantes de segundo de bachillerato y como $\mathrm{Hi}$ : El estado emocional incide en el rendimiento 
académico de los estudiantes de segundo de bachillerato. Mediante el proceso de verificación de hipótesis aplicando estadísticos pertinentes bajo el uso del SPSS (Statical Product and Service Solutions), se procede a la comprobación de la hipótesis partiendo de las siguientes fases:

$\checkmark$ Primera fase: (Planteamiento de las hipótesis de investigación).

$\checkmark$ Segunda fase: (Identificación del nivel de significancia)

$\checkmark$ Tercera fase: (Organización de datos y aplicación de pruebas de normalidad)

$\checkmark$ Cuarta fase: (Procesamiento de datos mediante SPSS, establecimiento de estadísticos acodes a los resultados obtenidos en las pruebas de normalidad)

$\checkmark$ Quinta fase: (Comprobación de hipótesis, descripción de correlación obtenida y decisión final)

Para establecer el nivel de significancia se procede a analizar que los valores presentados muestran un nivel de significancia menor a 0,05; para que exista una correlación entre ambas variables el nivel de significancia debe ser mayor a $0,05(\mathrm{P}<0,05)$. Una vez establecido este apartado se procede a desglosar las dimensiones de acuerdo con cada variable de estudio.

\section{Tabla 12}

Dimensiones del Test de Eysenck y de la Escala de Autoconcepto Académico (EAA)

\begin{tabular}{cll}
\hline \multicolumn{2}{c}{ Test de Eysenck Cuestionario para medir el nivel de Inestabilidad - Estabilidad emocional. } \\
\hline \multicolumn{2}{c}{ Inestabilidad Emocional } & Estabilidad Emocional \\
\hline$\bullet$ & Sentido de inferioridad & Autoestima \\
\hline$\bullet$ & Depresión & Felicidad \\
\hline$\bullet$ & Ansiedad & Calma \\
\hline$\bullet$ & Obsesividad & Indiferencia \\
\hline$\bullet$ & Dependencia & Autonomía \\
\hline & Hipocondría & Sensación de Salud \\
\hline & Sentido de Culpa & Sin culpabilidad \\
\hline Escala de Autoconcepto Académico (EAA) & \\
\hline & Rendimiento Académico \\
\hline & Autoeficacia \\
\hline & Autoconcepto \\
\hline
\end{tabular}

Fuente: Datos obtenidos de la aplicación de test en Google Forms

Una vez establecidas las dimensiones propuestas por cada instrumento se procede a procesar los datos mediante SPSS, se realizan las pruebas de normalidad pertinentes para establecer si los datos son normales. Como se observa en la tabla 13, la prueba de normalidad determina que para dos series de datos normales se debe aplicar el estadígrafo de Correlación de Pearson y para los datos que no son normales Rho de Spearman. 


\section{Tabla 13}

Pruebas de Normalidad Procesadas SPSS

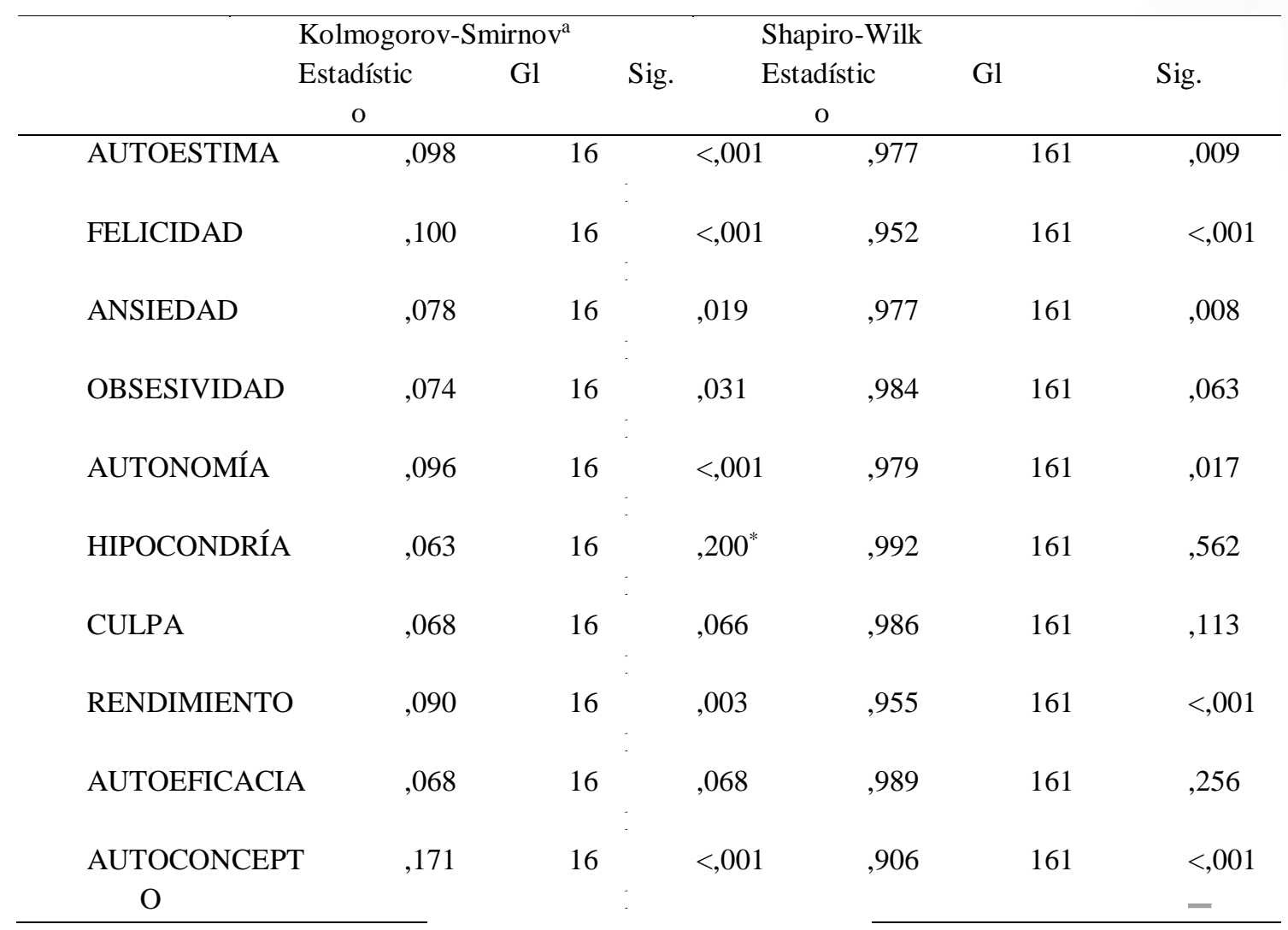

Fuente: Datos obtenidos SPSS

\section{Decisión}

Una vez obtenidos los resultados de correlación de las variables: Estado emocional y rendimiento académico. Se procede a concluir lo siguiente:

Con un margen de error de 0,05, el valor de Pc $<0,005 \mathrm{H} 0$ se obtiene mediante una aproximación significativa (P) igual (Sig. Bilateral 1,00). Los valores obtenidos en factores como: autoestima - inferioridad y rendimiento académico, autoestima inferioridad y autoeficacia, ansiedad - calma y autoeficacia, y culpa y ausencia de culpabilidad y autoconcepto académico, fueron correlacionados y se obtuvieron los valores pertinentes para rechazar la hipótesis nula Ho y aceptar la hipótesis alterna H1. Los valores demuestran que entre la dimensión Autoestima - Inferioridad y Autoeficacia el índice de correlación es de 0,75 lo que demuestra que la relación existente entre los mismos es fuerte y se comprueba $\mathrm{H} 1$ y se rechaza la hipótesis nula. Por otro lado, la relación existente entre autoestima - inferioridad y autoeficacia es media 0,65 y la significancia es menor a 0,05 por lo tanto, se rechaza la hipótesis nula y se acepta la hipótesis alterna. En el caso de la dimensión ansiedad - calma y autoeficacia se establece 
que el índice de correlación es de 0,51 por lo tanto existe una relación media entre las variables, además la significancia es menor a 0,05 por consiguiente, se rechaza la hipótesis nula y se acepta la hipótesis alterna. Por último, en las dimensiones sentido de culpa - ausencia de culpabilidad y autoconcepto académico se demuestra que el índice de correlación es 0,71 que indica que existe una relación fuerte entre las variables, en cuanto a la significancia se obtuvo un valor menor a 0,05 lo que indica que se rechaza la hipótesis nula y se acepta la alterna.

Sin embargo, Pc> 0,05 Ho, no se obtiene aproximaciones significativas en las dimensiones: Felicidad - Depresión y Rendimiento académico, ansiedad - calma y rendimiento académico, indiferencia - obsesividad, dependencia - autonomía, sensación de salud - hipocondría y sentimiento de culpa - ausencia de culpabilidad y rendimiento académico; en estos casos al ser la significancia mayor a 0,05, se acepta la hipótesis nula y se rechaza la alterna, es decir, el estado emocional no incide en el rendimiento académico. Además, la correlación establecida entre autoeficacia y depresión - felicidad, obsesividad - indiferencia, autonomía - dependencia, sensación de salud - hipocondría y sentimientos de culpa - ausencia de culpabilidad, se acepta la hipótesis nula que indica que el estado emocional no incide en el rendimiento académico.

Por último, la correlación establecida entre autoconcepto académico y autoestima inferioridad, ansiedad - calma, felicidad - depresión, indiferencia - obsesividad y sensación de salud - hipocondría denotaron valores mayores a 0,05 por lo tanto se acepta la hipótesis nula que indica que el estado emocional no incide en el rendimiento académico.

Tabla 14

Correlación: estado emocional y rendimiento académico

\begin{tabular}{|c|c|c|c|c|c|c|c|}
\hline & $\begin{array}{l}\text { Autoestima - } \\
\text { Inferioridad }\end{array}$ & $\begin{array}{l}\text { Felicidad- } \\
\text { Depresión }\end{array}$ & $\begin{array}{l}\text { Calma - } \\
\text { Ansiedad }\end{array}$ & $\begin{array}{l}\text { Indiferencia - } \\
\text { Obsesividad }\end{array}$ & $\begin{array}{l}\text { Autonomía - } \\
\text { Dependencia }\end{array}$ & $\begin{array}{l}\text { Sensación de } \\
\text { Salud - } \\
\text { Hipocondría }\end{array}$ & $\begin{array}{l}\text { Culpa-No } \\
\text { culpabilidad }\end{array}$ \\
\hline $\begin{array}{l}\text { Correlación de } \\
\text { Pearson }\end{array}$ & No aplica & No aplica & No aplica & 0,85 & No aplica & 0,69 & 0,91 \\
\hline $\begin{array}{l}\text { Correlación Rho } \\
\text { Spearman }\end{array}$ & 0,75 & $-0,49$ & 0,49 & No aplica & 0,25 & No Aplica & No aplica \\
\hline $\begin{array}{l}\text { Significancia } \\
\text { asintótica } \\
\text { (bilateral) }\end{array}$ & 0,02 &, 593 &, 590 & ,282 &, 750 &, 384 &, 250 \\
\hline Valor $\mathrm{P}$ & $<0,05$ & $>0,05$ & $>0,05$ & $>0,05$ & $>0,05$ & $>0,05$ & $>0,05$ \\
\hline
\end{tabular}

Fuente: Datos obtenidos SPSS 


\section{Tabla 15}

Correlación Estado emocional y Autoeficacia

\begin{tabular}{|c|c|c|c|c|c|c|c|}
\hline & $\begin{array}{l}\text { Autoestima - } \\
\text { Inferioridad }\end{array}$ & $\begin{array}{l}\text { Felicidad - } \\
\text { Depresión }\end{array}$ & $\begin{array}{l}\text { Calma - } \\
\text { Ansiedad }\end{array}$ & $\begin{array}{l}\text { Indiferencia - } \\
\text { Obsesividad }\end{array}$ & $\begin{array}{l}\text { Autonomía - } \\
\text { Dependencia }\end{array}$ & $\begin{array}{l}\text { Sensación de } \\
\text { Salud - } \\
\text { Hipocondría }\end{array}$ & $\begin{array}{l}\text { Culpa-No } \\
\text { culpabilidad }\end{array}$ \\
\hline $\begin{array}{l}\text { Correlación de } \\
\text { Pearson }\end{array}$ & No aplica & No aplica & No aplica & 0,44 & No aplica & 0,33 & 0,56 \\
\hline $\begin{array}{l}\text { Correlación Rho } \\
\text { Spearman }\end{array}$ & 0,65 & $-0,65$ & 0,51 & No aplica & $-0,11$ & No aplica & No aplica \\
\hline $\begin{array}{l}\text { Significancia } \\
\text { asintótica } \\
\text { (bilateral) }\end{array}$ & 0,03 & ,413 & 0,00 &, 582 & ,892 & ,974 &, 480 \\
\hline Valor de P & $<0,05$ & $>0,05$ & $<0,05$ & $>0,05$ & $>0,05$ & $>0,05$ & $>0,05$ \\
\hline
\end{tabular}

Fuente: Datos obtenidos SPSS

Tabla 16

Correlación: Estado emocional y Autoconcepto Académico

\begin{tabular}{llllllll}
\hline & $\begin{array}{l}\text { Autoestima } \\
\text { Inferioridad }\end{array}$ & $\begin{array}{l}\text { Felicidad }- \\
\text { Depresión }\end{array}$ & $\begin{array}{l}\text { Calma }- \\
\text { Ansiedad }\end{array}$ & $\begin{array}{l}\text { Indiferencia - } \\
\text { Obsesividad }\end{array}$ & $\begin{array}{l}\text { Autonomí - } \\
\text { Dependencia }\end{array}$ & $\begin{array}{l}\text { Sensación de } \\
\text { Salud - } \\
\text { Hipocondría }\end{array}$ & $\begin{array}{l}\text { Culpa - No } \\
\text { culpabilidad }\end{array}$ \\
\hline $\begin{array}{l}\text { Correlación de } \\
\text { Pearson }\end{array}$ & No aplica & No aplica & No aplica & 0,07 & No aplica & No aplica & No aplica \\
\hline $\begin{array}{l}\text { Correlación Rho } \\
\text { Spearman }\end{array}$ &,- 101 & $-0,76$ & 0,52 & No aplica & $-0,40$ & 0,49 & 0,71 \\
\hline $\begin{array}{l}\text { Significancia } \\
\text { asintótica } \\
\text { (bilateral) }\end{array}$ &, 203 &, 339 &, 514 &, 927 &, 617 &, 533 &, 002 \\
\hline \begin{tabular}{l} 
Valor de P \\
\hline
\end{tabular} & $>0,05$ & $>0,05$ & $>0,05$ & $>0,05$ & $>0,05$ & $>0,05$ & $<0,05$ \\
\hline
\end{tabular}

Fuente: Datos obtenidos SPSS

\section{Conclusiones}

- Por medio de la evaluación realizada con el Test de Eysenck se logró determinar que existe la relación directa con tendencias fuerte a moderada ente la Autoestima, la ansiedad y el rendimiento académico. Por otro lado, existe relación entre la ansiedad y la autoeficacia. Además, existe relación entre los sentimientos de culpa y el autoconcepto académico. Estos resultados son fundamentales para la comprobación de la hipótesis en varias dimensiones. De esta forma queda demostrado que algunas dificultades del estado emocional están asociadas con el rendimiento académico. Esto otorga pautas esenciales para procesos de intervención temprana previniendo la prevalencia de trastornos relacionados con la inestabilidad emocional. 
- A su vez, se determinó que el estado emocional general de los estudiantes de segundo de bachillerato es inestable menor a 30. Existen características marcadas de inestabilidad emocional que se caracteriza por la presencia de comportamientos asociados con sentimientos de inferioridad (16/30), tendencia moderada a la depresión (15/30), ansiedad (15/30), rasgos de dependencia (16/30), actitudes de hipocondría (12/30) y sentimientos de culpa (15/30). La evaluación permitió identificar a profundidad las dificultades del estado emocional, logrando explorar y obtener resultados base para implementar proyectos, talleres o actividades encaminadas al mejoramiento del estado emocional de los estudiantes.

- Por último, se identificaron los aspectos principales del rendimiento académico como la Autoeficacia, el Autoconcepto y el rendimiento. Los mismos que están en un rango bueno - regular. Es preciso enfatizar en el desarrollo de la autoeficacia, la capacidad de reconocer y confiar en las propias habilidades del sujeto para que logre un nivel de aprendizaje óptimo. Por otro lado, es necesario considerar que los DECES como unidades de apoyo en las instituciones educativas, puedan empoderarse acerca de temas de salud mental y desarrollar propuestas encaminadas al desarrollo de proceso de regulación emocional, toma de conciencia de las acciones, modificación conductas disruptivas, manejo de crisis emocionales, entre otros. Todo esto con la finalidad de evitar posibles dificultades que comprometan el rendimiento académico.

\section{Referencias bibliográficas}

Berlanga, A. (2008). La inestabilidad emocional. INVAL-Instituto Valenciano de Psicología, http://www.lasprovincias.es/valencia/20080614/salud/psicologiainestabilidad-emocional-20080614.html.

Boyle, G. J. (2016). Hans J. Eysenck and Raymond B. Cattell on intelligence and personality. Personality and Individual Differences, vol. 103, p. 40-47.

Brackett, M. \&. (2020). Emotional Intelligence Comes of Age. In Cerebrum: The Dana Forum on Brain Science (Vol. 2020). Dana Foundation.

Gallardo Vázquez, P. (2016). El desarrollo emocional en la educación primaria (6-12 años). Cuestiones pedagógicas, 18, 145-161.

García-Cerda, L., Rodríguez-Fernández, O., Betancourt-Galindo, R., Saldívar-Guerrero, R., \& Torres-Torres, M. (2003). Síntesis y propiedades de ferrofluidos de magnetita. Superficies y Vacío., 16(1), 28-31. 
Gracia, E. V.-S.-T. (2016). Análisis de las características de estabilidad emocional en hombres y mujeres. Psychologic, 59(2), 137-147.

Guajardo, E. S. (2015). Conocimiento y desarrollo emocional, desde el enfoque cognitivo, procesal sistémico. Límite. Revista Interdisciplinaria de Filosofía y Psicología, 1(12), 31-52.

Güemes-Hidalgo, M. C. (2017). Pubertad y adolescencia. ADOLESCERE Revista de Formación Continuada de la Sociedad Española de Medicina de la Adolescencia, 5(1), 07-22.

Hoffmann, J. D. (2020). Teaching emotion regulation in schools: Translating research into practice with the RULER approach to social and emotional learning. Emotion, 20(1), 105.

Melamed, A. F. (2016). Las teorías de las emociones y su relación con la cognición: un análisis desde la filosofía de la mente.

Meléndez, Y. C. (2016). Las emociones en el proceso de enseñanza-aprendizaje. Revista vinculando.

Mónaco, E. d.-C. (2017). Desarrollo de un programa de intervención para mejorar las competencias emocionales, el afecto positivo y la empatía en la adolescencia. Calidad de vida y salud, 10(1)., 10(1).

Navarro, R. E. (2003a). El rendimiento académico: concepto, investigación y desarrollo. Revista Iberoamericana sobre Calidad, Eficacia y Cambio en Educación, REICE.1(2).

Obando, C. J. (2017). El rendimiento académico: aproximación necesaria a un problema pedagógico actual. Revista Conrado, 13(58), 213-220.

Orellana, C. T. (2021). Bienestar Docente durante la Pandemia COVID-19: La Comparación entre Ecuador y Chile. Revista de Sociología, 325-347.

Pekrun, R. L. (2017). Achievement emotions and academic performance: Longitudinal models of reciprocal effects. Child development, 88(5), 1653-1670.

Pulido A \& Herrera Clavero, F. (2017). La influencia de las emociones sobre el rendimiento académico. Ciencias Psicológicas, 11(1), 29-39.

Ripoll, R. (2014). Fundamentos de Psicobiología. Cataluña: Editorial UOC.

Rovella, A. y. (2018). El Estudio Dimensional de las emociones. Aportes de la neurobiología. contextos de educación, 25. 
Schmidt, V. M. (2008). Autoconcepto académico en adolescentes de escuelas medias: presentación de un instrumento para su evaluación. Revista Iberoamericana de Diagnóstico y Evaluación-e Avaliação Psicológica. 1(25), 81-106.

Silva, C. B. (2014). Rendimiento académico y las dimensiones personal y contextual del aprendizaje socioemocional: evidencias de su asociación en estudiantes chilenos. Universitas Psychologica, 13(2), 627-638.

Valiente, C. S. (2012). Linking students' emotions and academic achievement: When and why emotions matter. Child development perspectives, 6(2), 129-135.

\section{$\lfloor$ Ciencia}


El artículo que se publica es de exclusiva responsabilidad de los autores y no necesariamente reflejan el pensamiento de la Revista Conciencia Digital.

\section{Ciencia
Digital
Edtatiol}

El artículo queda en propiedad de la revista y, por tanto, su publicación parcial y/o total en otro medio tiene que ser autorizado por el director de la Revista Conciencia Digital.
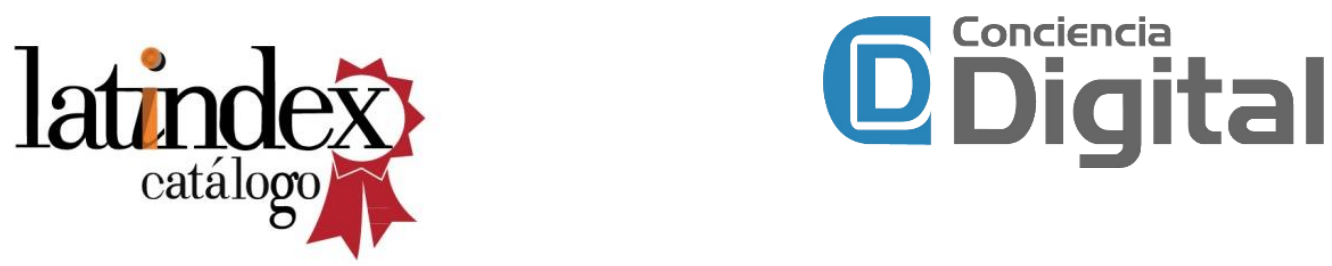

Indexaciones

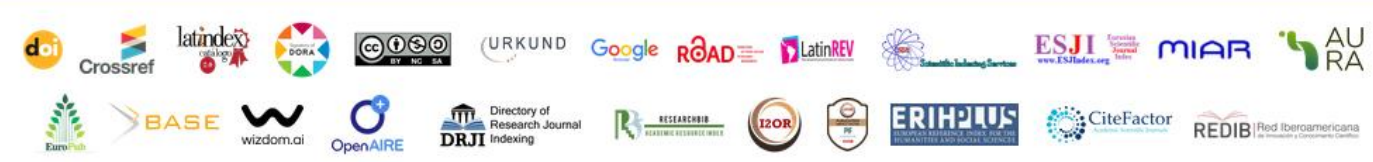

